\title{
Effects of centralized and local PV plant control for voltage regulation in LV feeder based on cyber-physical simulations
}

\author{
Theis Bo RASMUSSEN ${ }^{1}$ (D), Guangya YANG $^{1}$, Arne Hejde NIELSEN ${ }^{1}$, \\ Zhaoyang DONG
}

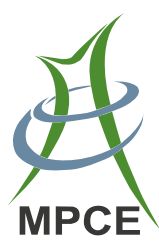

\begin{abstract}
In the modern power system, both local and centralized reactive power control strategies for photovoltaic (PV) plants, are proposed and compared. While local control improves the network security, it lacks the optimization benefits from centralized control strategies. Therefore, this paper considers the coordination of the two control strategies, depending on external impact from the weather system and consumer behavior, in a low voltage (LV) distribution feeder. Through modeling and simulation in an established real-time cyber-physical simulation platform, the LV network is evaluated with both local and centralized control. A set of boundaries for coordinating between the two strategies are identified, which can help network operators in deciding suitable control in different operating situations. Furthermore, the cyber-physical simulation platform, is used to study the impact of physical perturbations, i.e. changes in irradiance and consumption, and cyber disturbances, in form of communication channel
\end{abstract}

CrossCheck date:27 July 2018

Received: 29 November 2017/Accepted: 27 July 2018/Published online: 5 September 2018

(C) The Author(s) 2018

$\triangle$ Theis Bo RASMUSSEN

thras@elektro.dtu.dk

Guangya YANG

gyy@elektro.dtu.dk

Arne Hejde NIELSEN

ahn@elektro.dtu.dk

Zhaoyang DONG

joe.dong@unsw.edu.au

1 Center for Electric Power and Energy, Technical University of Denmark, Kgs. Lyngby, Denmark

2 School of Electrical Engineering and Telecommunications, University of New South Wales, Sydney, Australia noise, is evaluated for the control strategies. Results show how small and large disturbances in the cyber system affects the centralized control strategy optimizer performance.

Keywords Distribution Network, Voltage regulation, Photovoltaics, Cyber-physical system

\section{Introduction}

Through governmental subsidies and a general interest in converting to renewable sources of energy, both wind and solar based generation have experienced an increased level of penetration in the power system. From a low voltage (LV) distribution network perspective, countries like Denmark have seen a rise in the integration of roof-top photovoltaic (PV) plants at residential consumers. While the climate benefits from integrating renewables are obvious, the integration challenges the security of the power system [1].

The integrated generating units place additional stress on the network infrastructure, especially as voltage rise problems at nodes far away from the feeder root node. These problems occur due to the unidirectional power flow design of the radial feeders. This place a necessity for PV plant control methods, that can alleviate voltage rise problems $[1,2]$.

From an economic perspective, curtailment of PV generation is considered a last resort, which focus research on voltage regulation using reactive power control. Local control strategies utilize local measurements to decide on an appropriate power factor or reactive power set-point, and typically follow a droop-based control scheme with a defined droop ratio [1]. The definition of the droop ratio 
can be done through grid code specification [3, 4] or more advanced coordination-based approaches [5, 6]. Similar for all local control strategies is their ability to alleviate voltage rise issues, but also their limited capability to adjust to overall optimization since they follow a certain profile [7].

To achieve optimal utilization of power system infrastructure, minimizing the network losses or limiting the voltage variations, centralized methods based on optimization are proposed [7, 8]. The integration of such methods requires communication of network measurements, processing of the data, and ultimately communication of the controls. Therefore, the utilization of centralized control depends on the information and communications technology (ICT) infrastructure [9, 10].

When evaluating the performance of the centralized control strategies, it is important to consider the effects of disturbances in the acquisition, communication and command process, especially as centralized control tend to push the operation of the network towards vulnerable regions [7-9]. The investigation of cyber disturbances on centralized control strategies can be done using a cyber-physical simulation platform $[10,11]$. Such a platform can model and simulate the cyber and physical systems to show the relations between the two domains at different levels of complexity. For the evaluation of centralized control of PV plants in LV networks, the simulation platform must support optimization and real-time control, which emulates the processes in a control center. With a simulation platform capable of emulating a distribution network control center, both local and centralized control strategies can be simulated with the aim of coordinating their use during different operational situations.

In current literature, local and centralized reactive power control strategies, for residential PV plants, have been proposed and compared, but there is no literature supporting the decision-making process between the two strategies according to different operational scenarios $[1,5,7]$. Coordinating between the two control strategies enables the exploitation of the voltage rise alleviation capabilities from local control, and the overall optimality benefits from centralized control. Therefore, this paper proposes an identification of boundaries in the irradianceconsumption plane of a LV radial feeder, which serve as a base for coordination of centralized and local reactive power control of residential PV plants.

The intention with this coordination is to assist the network operator in the decision-making, and thereby ensure secure operation of LV networks in the cyberphysical system, without the shortcomings of each individual control strategy. The proposed coordination, therefore considers the risk of perturbations and disturbances in the cyber-physical system. It is based on extensive testing through cyber-physical modeling and real-time simulation of residential PV plants, using the Cigré European LV residential feeder as an example [12].

Results from the cyber-physical simulator show two zones for coordinating reactive power control, one area where the centralized control improves the operation of the network without jeopardizing the security of supply, and one region where local control is suggested. Furthermore, the simulations with cyber disturbances during centralized control show how artifacts in the communication channels can have a considerable impact of the performance of the control strategy.

The rest of the paper is outlined as follows. Section 2 presents the reactive power control strategies considered for coordination in this work. Section 3 discusses the simulation requirements for evaluating the control strategies, and describes the establishment of the real-time cyber-physical simulation platform. Section 4 presents the evaluation method for coordinating the control strategies from a cyber-physical perspective. Section 5 describes the considered case study LV feeder and test scenarios, Sect. 6 presents the simulation results and their analysis, and Sect. 7 concludes.

\section{Reactive power control strategies}

For local control of reactive power from PV plants, there exist different open loop approaches, e.g. constant power factor, constant reactive power, inverter power factor as a function of the active power injection, $\cos \varphi(P)$, and inverter reactive power transfer as a function of the terminal node voltage magnitude, $Q(V)$ [3].

Besides the proposal of different strategies, current literature focus on finding appropriate droop ratios for different methods [6, 13-15], and on coordinating these parameters in a specific feeder [5]. Since the focus of the current study is to identify boundaries for the purpose of coordinating between local and centralized control, a grid code specified implementation of $\cos \varphi(P)$ control is considered.

The chosen droop rate considered for all the PV plants in this work, is based on the grid codes from the Danish transmission system operator [4]. This strategy is shown graphically in Fig. 1.

Local reactive power control has static characteristics and open loop, as it strictly follows the reactive power control plan. Oppositely, centralized control comes with added capabilities of optimizing the operation of the distribution network towards a desired objective, i.e. minimizing voltage deviation, reactive power flows, active power losses, etc. $[2,7,8]$. 


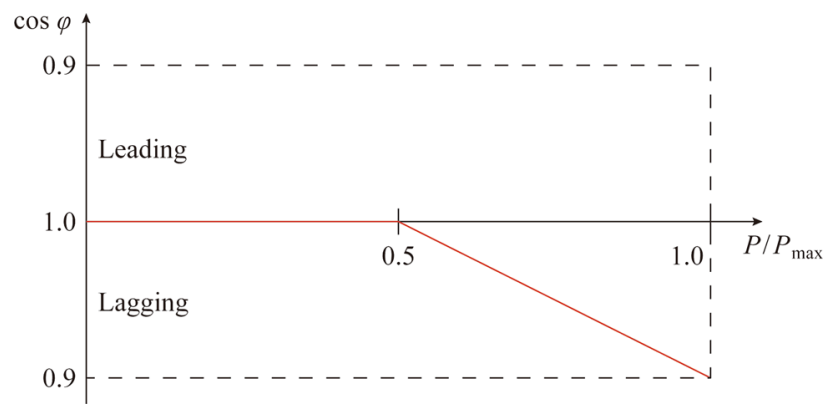

Fig. 1 Reactive power control of PV plant using local control according to Danish grid codes [4]

Since the focus of this work is to investigate the local and centralized control coordination, the optimization problem of the centralized controller is formulated with the objective to minimize the active power losses through identification of optimal inverter reactive power set points. By minimizing the active power losses in the distribution network, the total amount of active power being distributed is minimized, which allows better utilization of the power system infrastructure. The mathematical model of the optimization problem is described by the objective function in (1) and the constraints in (2)-(7).

$\min z=\sum_{n=1}^{N_{n}}\left(P_{n}^{\text {gen }}-P_{n}^{\text {load }}\right)$

s.t.

$P_{n}^{\text {gen }}-P_{n}^{\text {load }}=\left|V_{n}\right| \sum_{k=1}^{N_{n}}\left|Y_{n k}\right|\left|V_{k}\right| \cos \left(\theta_{n}-\theta_{k}-\delta_{n k}\right) \quad \forall n \in N_{n}$

$Q_{n}^{\text {gen }}-Q_{n}^{\text {load }}=\left|V_{n}\right| \sum_{k=1}^{N_{n}}\left|Y_{n k}\right|\left|V_{k}\right| \sin \left(\theta_{n}-\theta_{k}-\delta_{n k}\right) \quad \forall n \in N_{n}$

$\left|S_{l}^{\text {flow }}\right| \leq S_{l}^{\max } \quad \forall l \in N_{l}$

$P_{n}^{\min } \leq P_{n}^{\text {gen }} \leq P_{n}^{\max } \quad \forall n \in N_{n}$

$Q_{n}^{\min } \leq Q_{n}^{g e n} \leq Q_{n}^{\max } \quad \forall n \in N_{n}$

$V^{\min } \leq\left|V_{n}\right| \leq V^{\max } \quad \forall n \in N_{n}$

where $z$ is the objective function; $N_{n}$ is the total number of nodes; $P_{n}^{\text {gen }}$ and $Q_{n}^{\text {gen }}$ are the active and reactive power output from the generating unit at node $n ; P_{n}^{\text {load }}$ and $Q_{n}^{\text {load }}$ are the active and reactive power consumption at node $n$; $\left|V_{n}\right|$ is the voltage magnitude at node $n ;\left|Y_{n k}\right|$ is the magnitude of admittance between nodes $n$ and $k ; \theta_{n}$ is the voltage angle at node $n ; \delta_{n k}$ is the angle of admittance between nodes $n$ and $k ;\left|S_{l}^{\text {flow }}\right|$ is the apparent power flow in line $l ; S_{l}^{\max }$ is the maximum apparent power flow in line $l$; $P_{n}^{\min }$ and $P_{n}^{\max }$ are the minimum and maximum active power from generator at node $n ; Q_{n}^{\min }$ and $Q_{n}^{\max }$ are the minimum and maximum reactive power from generator at node $n$; and $V^{\min }$ and $V^{\max }$ are the minimum and maximum voltage magnitude at all nodes, assumed to be 0.9 p.u. and 1.1 p.u., respectively.

When solving the optimization problem in (1)-(7), the active power generation of the PV plants is assumed uncontrollable. The reactive power capabilities of the PV plants are assumed limited in (6), by a power factor between 0.9 lagging and 0.9 leading.

From a research perspective, local control strategies are easier to investigate as the control can be modelled directly in a power system simulator. For centralized control strategies, the power system simulator must be connected to computational software, capable of calculating the optimal power flow (OPF) and finding the optimal solution to the optimization problem in (1)-(7).

\section{Real-time cyber-physical simulation platform}

Numerous cyber-physical simulation platforms are proposed in current literature, with different focus and levels of complexity $[11,16]$. The diversity of simulation platforms is due to the specific focus of studies, which affect the establishment of the platforms. Furthermore, while real-time power system simulators can be purchased and installed from manufacturers, current cyber-physical simulators are usually a combination of different software and hardware components. These components are connected to enable specific studies in different areas of power system research, e.g. transmission, distribution, microgrids, etc.

To establish a cyber-physical simulation platform, it is therefore important to define the study specific requirements and identify appropriate software and hardware components. In this work, there are five main requirements. The platform should, (1) be capable of solving the optimization problem defined in (1)-(7), (2) simulate a LV distribution network with high penetration of PV, (3) allow local control strategy implementation for the PV plants, (4) enable switching between different control strategies, and (5) represent a completely controllable cyber network.

With the list of requirements, an appropriate power system simulator would be the real time digital simulator (RTDS), as it can simulate a LV distribution network and allows implementation of local control strategies. The RTDS is a combination of hardware and modeling software, and has been extensively used in the formulation of cyber-physical simulation platforms [11, 16-18]. The RTDS allows real time external communication of simulated values through GTNETx 2 cards. These cards are installed with firmware that correspond to different 
protocols, such as PMU, DNP3 and IEC 61850 sampled values.

The RTDS is not capable of performing the optimization needed in the centralized PV control strategy, meaning it must be connected to additional software components. The MATLAB software can provide the needed optimization capabilities, and allow customization of the mathematical problem and the use of different solvers. Communication of simulation results and control signals between RTDS and MATLAB is possible by running the RTDS simulation software, called Runtime, as a TCP server [19]. The Runtime software is used to control and monitor the simulations conducted by the RTDS and contains different meters and actuators.

When running the Runtime software as a TCP server, its meters and actuators are visible from MATLAB, which enables direct control of the RTDS simulation through MATLAB [19]. However, the acquisition of data is more complicated as MATLAB must encode the Runtime meter data to a specific syntax, and afterwards decode the value to enable processing. Therefore, an alternative way of data acquisition from RTDS to MATLAB is considered.

From current literature on cyber-physical simulation platforms, the acquisition of data from RTDS can be done through an open protocol communication (OPC) server $[17,18]$. These servers are commonly used in industrial sites to manage the translation of data from different meters to a common protocol [20]. An OPC server can therefore directly communicate with the GTNETx2 card of the RTDS. Furthermore, MATLAB provides an OPC toolbox, making communication between the two components simple to establish.

The main argument against using the OPC communication channel for control signals is due to the limited computational resources of the RTDS, and the high relative high requirements for enabling controls through the OPC server.

The last requirement for the simulation platform involves the representation of the cyber-system. In different simulation platforms, communication system simulators, e.g. OPNET and NS-3, or hardware is used [11, 16-18]. While these give a realistic representation of the cyber-system and the specific protocols, they have limited controllability. For the current study focus, the platform should allow full observability of the network, and a representation of cyber disturbances, e.g. communication noise. Therefore, the cyber-system is emulated in the MATLAB software through the control of noise added after the acquisition of simulation results and before sending control signals to RTDS.

Compared to current literature, the established platform is similar to the platform proposed in [18], although with a simplified cyber-system representation and the use of
MATLAB instead of a hardware implementation of the control center. Likewise, the platform proposed in [17] differs from the one used in this study by the cyber-system representation and the use of Python to represent the control center.

When running, the cyber-physical simulation platform executes as illustrated by the flowchart in Fig. 2. The flowchart is split in a physical part, representing how control actuation done in Runtime are continuously recognized by the representation of the physical system in the RTDS and how the RTDS enables the external communication of measurements through the GTNETx 2 card.

The cyber system is then executed according to the DNP3 protocol and the user-defined execution. With the DNP3 protocol, values are only updated when their change is larger than a predefined threshold value [21]. Whenever requested, MATLAB can read measurements from the OPC server, update the parameter in the optimization problem constraints in (5)-(6), find the optimal solution through OPF, and send new control commands to the PV plants in the test grid.

\section{Evaluation of control strategies in cyber- physical environment}

To evaluate the cyber-physical nature of the power system, and how it affects the usage of both local and centralized reactive power control, different physical perturbations and cyber disturbances are considered.

In a LV feeder, considering faults on equipment most likely interrupts services as controls are limited. Instead, perturbations in the physical system considered in this work are based on the impact from neighboring systems, such as the weather system and the human interactions

The interdependency of the cyber-physical system indicates the necessity for analyzing not only how perturbations in the physical system affect the physical system, but also how cyber disturbances affect the physical system. Additionally, one could investigate how cyber disturbances and physical perturbations affect the cyber system, however, this requires a more detailed and realistic

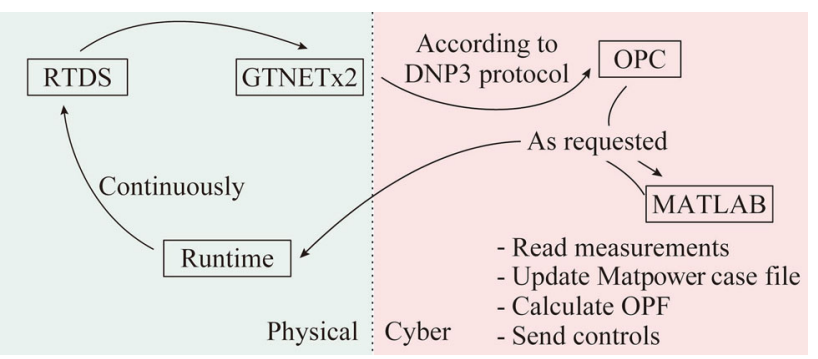

Fig. 2 Cyber-physical simulation platform execution flowchart 
representation of the ICT infrastructure, which is outside the scope for the current study.

While perturbations in the physical system affect the feeder when utilizing local and centralized control, cyber disturbances will have a smaller impact on local control since it is a continuous process. A single occurrence of error will therefore quickly be erased compared to the centralized control, where the control is only executed in a discrete fashion.

Cyber disturbances that can affect the physical system during centralized control, can be split into three areas. Errors occurring (1) in the process of acquiring measurements from the sensor and sending them to the centralized controller, (2) within the calculation of control commands, and (3) in the process of communicating and performing the commands send by the centralized controller.

In modern power systems, the use of state estimators for reducing the inclusion of measurement error is a wellknown practice for improving the accuracy of the input to further processing, such as the centralized controller $[22,23]$. The performance of the state estimator depends on the algorithm and the degree of freedom, therefore, different situations of error in the data acquisition, will be investigated in this study.

Since the occurrence of noise and error in the measurement acquisition and communication channels are possible due to the imperfections of the ICT infrastructure [24-27], a situation where control signals from the central controller are subject to error is also considered.

The analysis of the perturbations in the physical system and cyber disturbances is done by running the cyberphysical simulation platform while activating different scenarios, one at a time. The impact of these scenarios is evaluated through an analysis of the effects in voltage magnitude and active power losses. The network losses are used as a performance indicator as they are minimized in the centralized control strategy

The scale of impact from the scenarios depends on the power system feeder and the operational conditions considered. To scope the investigation, a Cigré European LV feeder model is chosen and modified to represent a future scenario, furthermore, a set of operational test scenarios have been formulated in terms of environment operation and component focus.

\section{Case study LV feeder}

The original Cigré LV feeder is presented in [12] and illustrated in Fig. 3a. To identify the coordination between local and centralized reactive power control of PV plants in the LV feeder, through simulation in the established cyber-

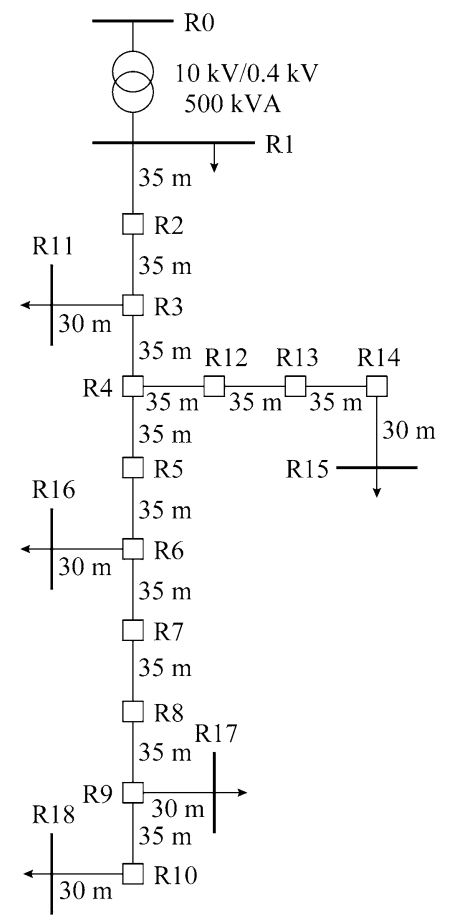

(a) Original grid

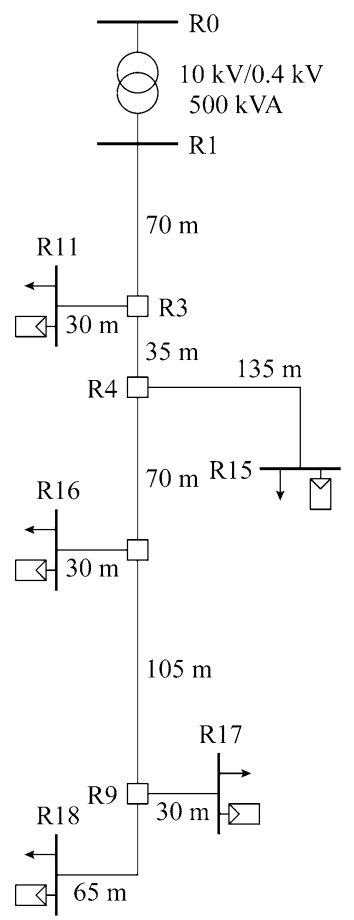

(b) Modified grid
Fig. 3 Original and modified single line diagrams of the Cigré European LV residential feeder [12]

physical simulation platform, the Cigré feeder has been modified in four ways.

1) The load connected to node R1 in Fig. 3a is removed and its demand is distributed among the rest of the loads. This gives higher requirements of power transfer and represents a feeder in an urban area of a large city with large building blocks connected to each load-point. The peak consumption of each load in the modified grid is given in Table 1 .

2) PV plants have been implemented at each of the load points, as shown in Fig. 3b, representing a scenario where each building block has invested in distributed generation. The installed PV capacity at each building block is given in Table 1.

Table 1 Modified Cigré LV feeder node peak consumption and generation

\begin{tabular}{lcc}
\hline Node & Load $(\mathrm{kVA})$ & PV $(\mathrm{kW})$ \\
\hline R11 & 55 & 15 \\
R15 & 92 & 42 \\
R16 & 95 & 42 \\
R17 & 75 & 21 \\
R18 & 87 & 42 \\
Total & 404 & 162 \\
\hline
\end{tabular}


3) Due to the resource limitations of RTDS, the cables in the original grid are aggregated to remove the nodes with zero current injection as shown in Fig. 3b.

4) The cables connecting R1 to R3 and R4 to R6 have been reinforced by implementing parallel lines to allow the higher transport of power in the distribution network.

All other grid details are as described for the original Cigré European LV residential feeder described in [12].

To choose the operational conditions of the power system environment, hourly data of irradiance for Copenhagen, Denmark (CPH) of the year 2005 [28], and hourly consumption data from apartments in Denmark in 2012 [29] is found.

To give an indication of the occurrence of the combinations of load and irradiance levels in $\mathrm{CPH}$, the gathered irradiance data have been rounded to nearest $10 \mathrm{~W} / \mathrm{m}^{2}$, and the gathered consumption data has been normalized to the largest consumption in the year and rounded to nearest $1 \%$ loading level. A heat map has been created as shown in Fig. 4 by finding the corresponding point in the irradianceconsumption plane for each hour in a year.

The current normal representation in Fig. 4 will change in a future scenario as new and renovated buildings have higher energy efficiency, which in general will lower the consumption of electricity for heating and cooling, and thereby shift the normal operating consumption towards zero. Besides having higher energy efficiency, buildings in the future will more likely be cost efficient as well. This means that the penetration of household PV plants increases. The irradiance scale in Fig. 4 can be considered as the percentage from 0 to $100 \%$ of rated $\mathrm{PV}$ power, as PV penetration increase in the future, the normal operating irradiance is shifted towards the right.

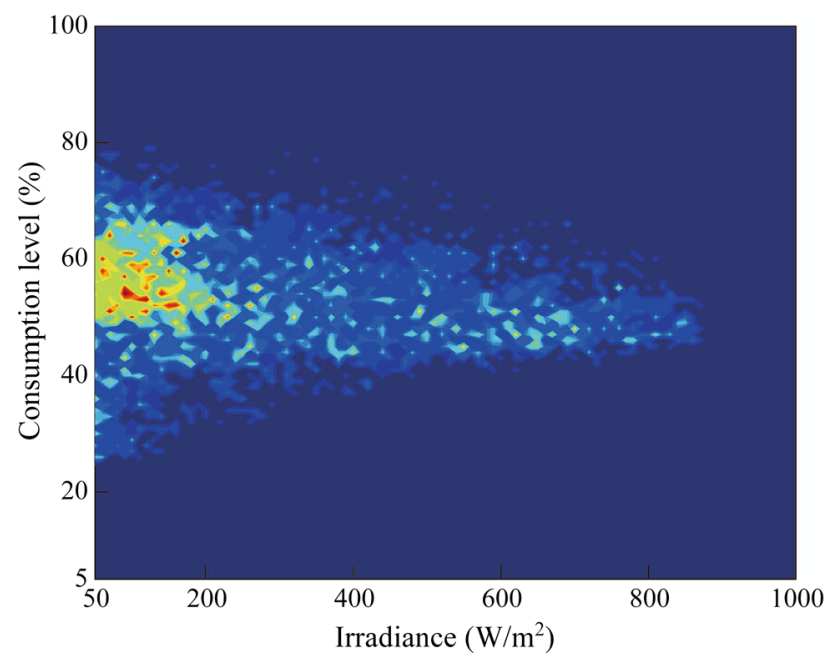

Fig. 4 Heatmap of irradiance and consumption data in Copenhagen based on hourly data $[18,19]$

\subsection{Test scenarios}

The first test scenario compares the two reactive power control strategies while the system is not exposed to any disturbances. The LV feeder is simulated in every possible operating situation in the irradiance-consumption plane and the results are evaluated from a voltage magnitude and network loss perspective.

In the second test scenario, the system is exposed to perturbations in the physical system, specifically as changes in consumption and irradiance level at node R15. Again, the voltage magnitude and network loss results are used to evaluate the effects of both reactive power control strategies. Based on the first two test scenarios, a set of guidelines for when to use local and centralized control, for the specific feeder in question, is formulated as the identified boundaries in the irradiance-consumption plane.

The third, fourth and fifth test scenarios then investigates the impact of cyber system disturbances on the centralized control strategy. The LV feeder is simulated in three different operating situations and exposed to noise in the data acquisition and command signal communication channels. The results of the simulations are evaluated, based on the effects on the optimizer performance, network losses, and voltage magnitude of the most vulnerable node.

\section{Results}

Simulations of the modified feeder in all possible combinations of the irradiance-consumption plane shown in Fig. 4, are done using the cyber-physical simulation platform. Specifically, the grid is simulated with a consumption level of $5 \%$ to $100 \%$, in steps of $5 \%$, and irradiance of $50 \mathrm{~W} / \mathrm{m}^{2}$ to $1000 \mathrm{~W} / \mathrm{m}^{2}$ in steps of $50 \mathrm{~W} / \mathrm{m}^{2}$. The resulting loss comparison is shown in Fig. 5 where the network losses as a function of irradiance level is plotted for four different consumption levels for both central $D_{C}$, solid lines, and local $D_{L}$, dashed lines, control.

Comparing the effect of the control strategies on the network losses in the feeder, clearly show a large difference when the irradiance approaches $1000 \mathrm{~W} / \mathrm{m}^{2}$, corresponding to rated PV plant operation. This is due to the inductive nature of the chosen reactive power control plan used in the local control strategy and shown in Fig. 1. At lower irradiance levels, the loss minimization from using centralized control, reduces with the reduced reactive power from the PV plants. In Fig. 5 the crosses represent the smallest irradiance level at which centralized control gives $1 \mathrm{~kW}$ less losses than local control.

An interesting observation in Fig. 5, is that there is an optimal PV generation for each loading level where the losses are minimal. This corresponds to the situation where 


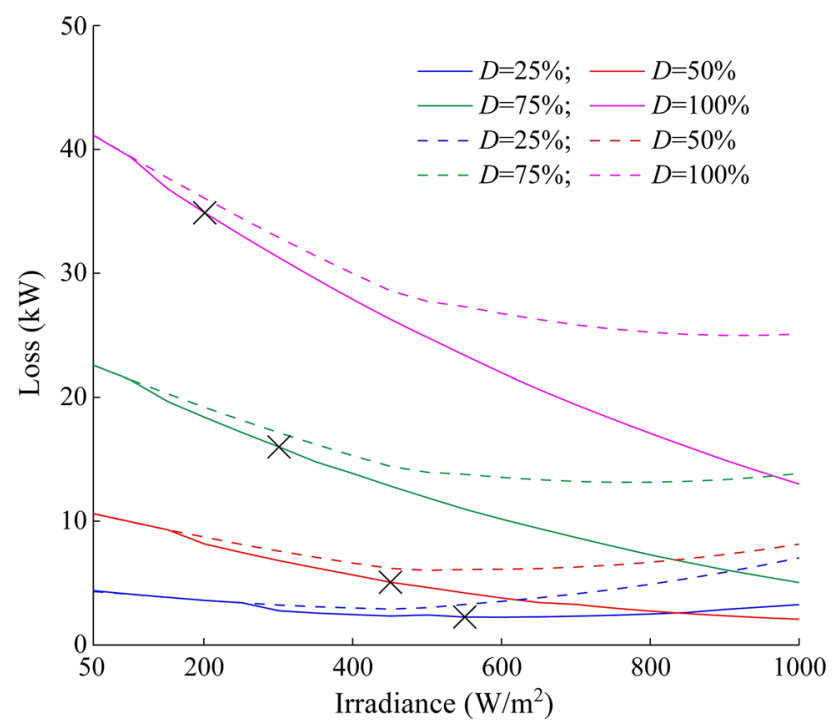

Fig. 5 Active power losses in the modified LV feeder using central $D_{C}$, solid lines, and local $D_{L}$, dashed lines, control, for different consumption and irradiance levels (Crosses show irradiance level where centralized control has $1 \mathrm{~kW}$ less losses than local control)

the consumption is mainly supplied by local PV, which minimize the amount of power flows in the grid.

The results from the simulations are further used to compare how the voltage magnitude is affected by local and centralized reactive power control. Theoretically, the highest voltage magnitude at any of the nodes in the modified feeder would happen in the bottom right corner of the irradiance-consumption plane, where consumption is low, and irradiance is high. The node in the modified LV feeder experiencing the highest voltage magnitude during low consumption and high irradiance, is found to be R15.

To investigate the voltage vulnerability of the system during normal operational situations, the heatmap of irradiance and consumption in Fig. 4 is layered with contour lines representing the node R15 voltage magnitude for each operational scenario. Two sets of contour lines are included in Fig. 6, the solid lines showing the results during centralized control, and dashed lines showing the results during local control.

From Fig. 6 clearly the voltage magnitude is most vulnerable during high irradiance and low consumption, in fact the voltage limit of 1.1 p.u. is exceeded during this operation with centralized control. Furthermore, the dashed contour lines show that during local reactive power control, the node R15 voltage magnitude will not exceed 1.1 p.u.. This clearly emphasizes the necessary tradeoff between voltage vulnerability and loss minimization.

Based on the heatmap for irradiance and consumption for $\mathrm{CPH}$, the local control strategy will at most result in 1.04 p.u. at node R15, while the centralized control can

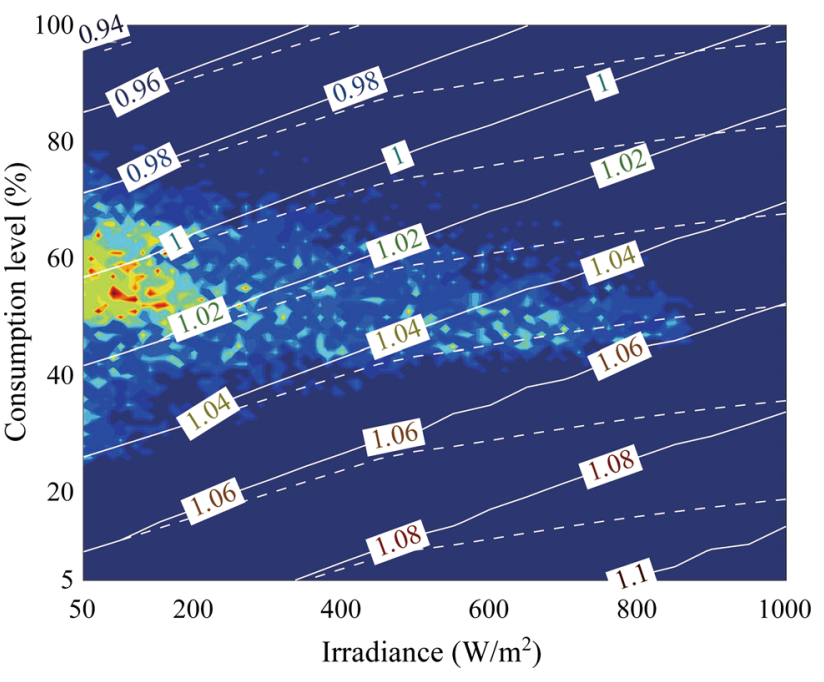

Fig. 6 Irradiance and consumption heatmap for normal operation in Copenhagen, with contour lines representing the voltage magnitude of node R15 during centralized control, solid line, and local control, dashed line

reach 1.06 p.u.. This is not exceeding the voltage magnitude limit of 1.1 p.u.. However, in a future scenario, the operating area could move towards the vulnerable region as houses become more energy and cost efficient and PV penetration increases.

\subsection{Perturbations in physical system}

Besides the long-term changes affecting the operational situation, the occurrence of perturbations in the physical system can be interpreted as shifting the operational area in the irradiance-consumption plane. This interpretation is valid under the assumption that the effects are only analyzed during static operation.

From the results in the first test scenario, the feeder experience overvoltage during high irradiance and low consumption when being centrally controlled. Therefore, the effect of physical perturbations is studied firstly during current normal operation of $\mathrm{CPH}$, which is highlighted in the blue area of Fig. 7, and secondly during more vulnerable operation, highlighted by in the yellow area of Fig. 7 . In both studies, the possible situations due to exposure of physical perturbations are included.

For each operational scenario, changes in consumption of 5\%, $10 \%$ and $20 \%$, negative and positive, and changes in irradiance of $50 \mathrm{~W} / \mathrm{m}^{2}, 100 \mathrm{~W} / \mathrm{m}^{2}$ and $200 \mathrm{~W} / \mathrm{m}^{2}$, negative and positive, are considered. The changes in the operational situation caused by the physical perturbations are represented in Fig. 7 in the green and the red areas for normal and vulnerable operation, respectively.

The physical perturbations are simulated in the cyberphysical simulation platform while controlling the PV 


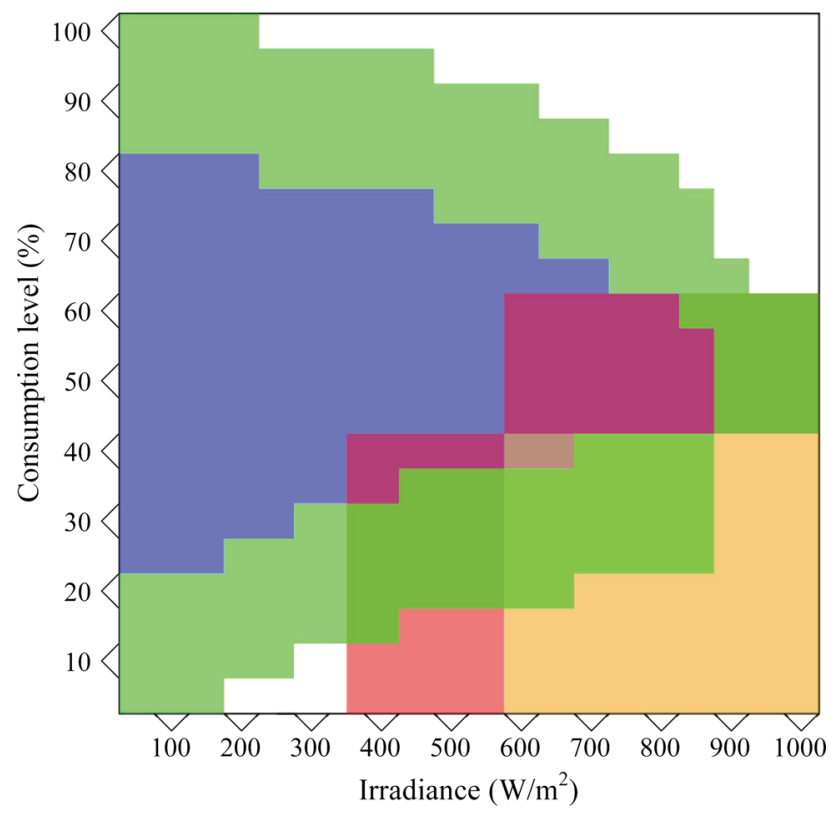

Fig. 7 Irradiance-consumption plane with the normal operation of Copenhagen in blue, including the area of operation during physical perturbations, in green, and a vulnerable operating area, in yellow, with matching physical perturbation area, in red

plants through the two control strategies. During local control, the system is simulated for a couple of seconds in each of the situations in the blue and yellow areas of Fig. 7, and exposed to each of the physical perturbations returning results representing the system operation in the blue, green, yellow and red areas of Fig. 7.

For the centralized control strategy, the reactive power is optimized at the situations in the blue and yellow areas of Fig. 7. After one second of simulation, the system is exposed to a perturbation in the physical system and simulated for further four seconds. Then the reactive power is optimized again, and the system is simulated for an additional five seconds. This gives an opportunity to analyze the impact of physical perturbations pre- and postoptimization.

The simulation results showed that in any of the normal operating situations, the system will survive the perturbations in the physical system considered, as none of the green areas in Fig. 7 cause overvoltage according to Fig. 6. For the vulnerable operating situation, the simulation results for $5 \%$ consumption and exposure to a $20 \%$ consumption increase, and a $200 \mathrm{~W} / \mathrm{m}^{2}$ irradiance, increase and decrease, are shown in Fig. 8.

In both plots of Fig. 8 the solid lines represent the simulation results during centralized control after a perturbation and before the optimizer has reacted to the new operational situation. The dashed lines represent the post perturbation and optimization results and the dotted lines represent local control.

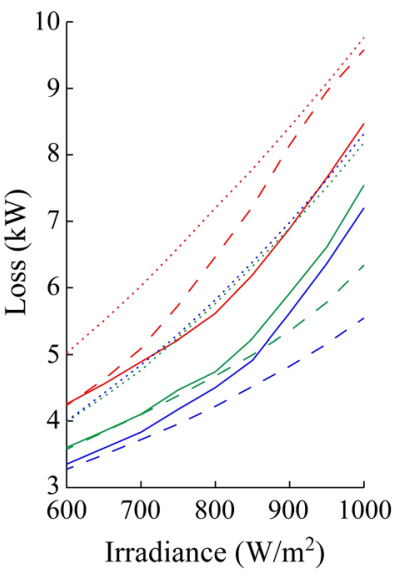

(a) Network losses

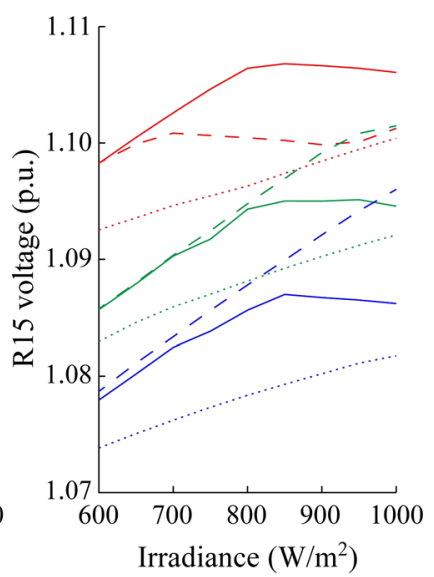

(b) Bus R15 voltage magnitude

$$
-+\mathrm{D} ;-+\mathrm{G} ;--\mathrm{G}
$$

Fig. 8 Results from simulating physical perturbations during 5\% consumption operating situation represented by the network losses and the node R15 voltage magnitude

The system losses in Fig. 8a as a function of irradiance show that at high irradiance and low consumption, a consumption increase, $+\mathrm{D}$, and irradiance decrease, $-\mathrm{G}$, will increase the losses until the reactive power has been optimized according to the new operational situation. At $1000 \mathrm{~W} / \mathrm{m}^{2}$, the pre-optimization losses nearly reach the local control system losses.

An irradiance increase, $+\mathrm{G}$, will have the opposite effect as the pre-optimization losses are lower than the post-optimization losses. The reason is clearly visible in Fig. $8 \mathrm{~b}$ where the irradiance increase causes the pre-optimization node R15 voltage magnitude to violate the $1.1 \mathrm{pu}$ voltage limit. The optimizer then lowers the voltage as seen by the post-optimization voltage magnitude, which increase the system losses. This observation shows the importance of caution when choosing an appropriate timeperiod between running the optimizer.

\subsection{Voltage regulation strategy coordination}

Through the analysis of the effects of local and centralized PV plant reactive power control, a set of guidelines for when to use local and centralized reactive power control of the PV systems in the modified LV feeder in Fig. 3b are established from a network loss minimization and voltage stability perspective.

The guidelines for coordinating the two control strategies are represented in Fig. 9, where the blue color represents areas to use local control and the orange color shows areas to use centralized control.

First observation, marked in Fig. 9, is made from the direct comparison between the two strategies during no 


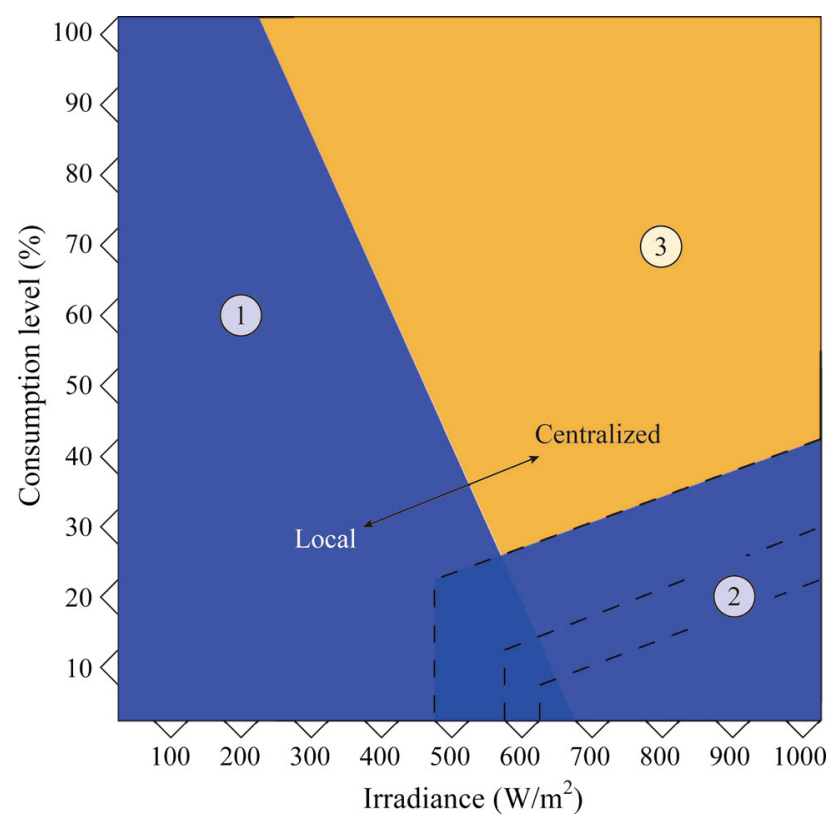

Fig. 9 Guidelines for operating the PV systems with local, blue area, and centralized, orange area, reactive power control

perturbations in the physical system. Here the results in Fig. 5 show that for each consumption level, the irradiance point where losses are significantly lower during centralized compared to local control. As an assumption for this work, a loss reduction of $1 \mathrm{~kW}$ is chosen as the point of using centralized control instead of local.

The second observation is based on the analysis of the impact from changes in consumption and irradiance level within the vulnerable operating area of Fig. 7 . Here the perturbations could cause overvoltage in node R15, especially during low consumption and high irradiance. Therefore, the high voltage vulnerability area in the bottom right corner of the irradiance-consumption plane is marked in blue in Fig. 9. Here three dashed lines represent the severity of physical perturbations, the system should be able to resist, with the largest to smallest areas representing a consumption change of $20 \%, 10 \%$ and $5 \%$, and an irradiance change of 200,100 and $50 \mathrm{~W} / \mathrm{m}^{2}$, respectively.

While the local control strategy offers security against overvoltage and decrease the utilization of communication, centralized control is recommended in the orange area of Fig. 9, as it can substantially decrease network losses without jeopardizing the voltage magnitudes during perturbations in the physical system. Utilizing centralized control however, entails a vulnerability towards disturbances in the cyber system, the effects of which are evaluated in the following section.

\subsection{Centralized control during cyber disturbances}

Through utilizing the cyber-physical simulation platform established in this work, the effects of three different cyber disturbances are investigated and analyzed:

1) The occurrence of noise in the acquisition and communication of observations in the physical system, of zero mean and $0.5 \%, 1 \%$ and $2 \%$ standard deviation.

2) The substitution of one or two of the acquired measurements by zeros. This scenario represents an extreme case where the state estimator fails and allow zero-readings as input to the centralized controller [30]. While this situation is unlikely, it is important to consider extreme scenarios when investigating the network security.

3) The occurrence of noise in the communication and interpretation of command signals, of zero mean and $0.5 \%, 1 \%$ and $2 \%$ standard deviation.

For each of the cyber disturbances, the LV feeder is simulated with centralized control in three different operating situations, each representing one of the three areas in Fig. 9.

1) $60 \%$ consumption and irradiance of $200 \mathrm{~W} / \mathrm{m}^{2}$.

2) $20 \%$ consumption and irradiance of $900 \mathrm{~W} / \mathrm{m}^{2}$.

3) $70 \%$ consumption and irradiance of $800 \mathrm{~W} / \mathrm{m}^{2}$.

When noise is introduced in the measurement acquisition part of the cyber system, the immediate effect will be on the performance of the optimizer. The investigation of the effects of the first cyber disturbance for each of the three operating situations is therefore done by comparison of the network losses (1) calculated by the optimizer using the original, correct, measurements, (2) calculated by the optimizer using measurements subject noise with the three different magnitudes of standard deviation, (3) calculated by the RTDS in the cyber-physical simulation platform, and (4) found from the first test scenario for the operating situations during local control.

Figure 10a, b and c, show the network losses, sorted ascendingly, for the three operating situations from minimum to maximum, where a standard deviation of $0.5 \%, 1 \%$ and $2 \%$ is assumed in simulations 1 to 200,201 to 400 and 401 to 600 , respectively.

The effects of measurement noise are clearly visible in Fig. 10a, where the network losses calculated by the optimizer using the original measurements is constant, and the losses calculated using noisy measurements deviates substantially as a function of the magnitude of the noise standard deviation. The noise therefore affects the optimizer to believe that it either can operate with fewer network losses, or must operate at higher network losses. 


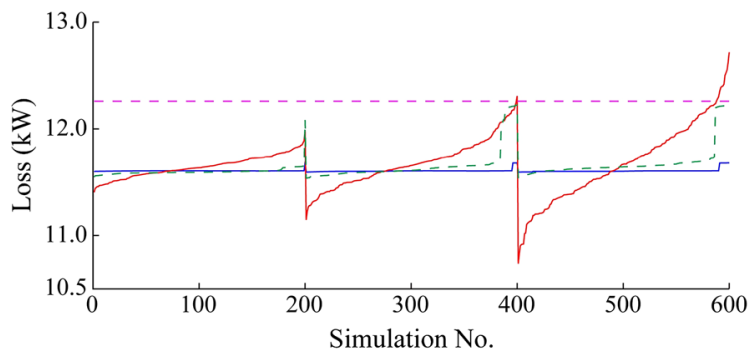

(a) Situation 1: 60\% consumption and $200 \mathrm{~W} / \mathrm{m}^{2}$ irradiance

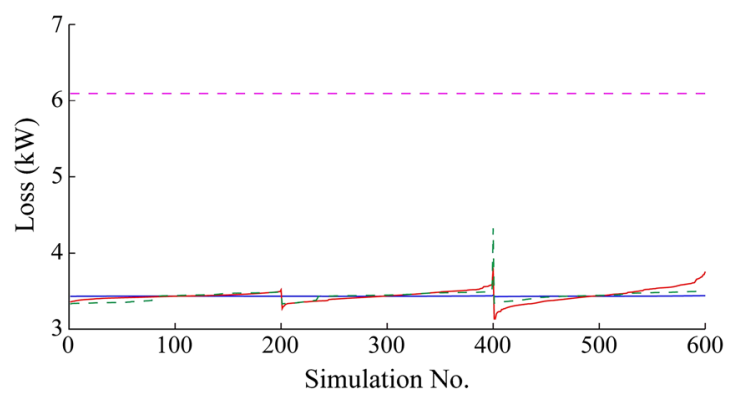

(b) Situation 2: 20\% consumption and $900 \mathrm{~W} / \mathrm{m}^{2}$ irradiance

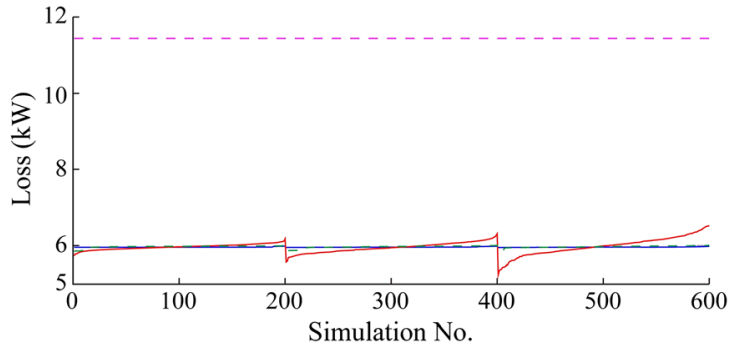

(c) Situation 3: 70\% consumption and $800 \mathrm{~W} / \mathrm{m}^{2}$ irradiance

- Optimizer (original); — Optimizer (noise); - - RTDS; - - - Local control

Fig. 10 Effects of small noise in the measurement acquisition channel during three different operating situations with centralized reactive power control

The difference between the optimizer calculated network losses and the actual losses observed in the RTDS are of cause due to the noise in the measurements which distorts the observability of the physical system. The severity of the impact from noise in measurements, from a network loss minimization perspective, can be analyzed by comparison of the RTDS observed losses and the previously calculated network losses during local control for the first operating situation. At a low noise magnitude, only a single simulation caused network losses close to the expected losses from local control. However, at 1 and $2 \%$ standard deviation more than 10 of the simulations, equal to $5 \%$, caused network losses only marginally different from what was found during local control.

By comparison of the effects from measurement noise in the three different operating situations, clearly the first situation is most affected, while the two other situations are only marginally affected. The effect is based on the influence of the PV plants in the system and the gradient in the network losses caused by changing the power factor setpoints.

As the nonlinear optimization problem is solved using the FMINCON algorithm, the optimal solution is found by initializing the algorithm with the noisy measurements and move in the direction of the largest negative gradient of the network losses. During high irradiance and low noise levels, the algorithm will therefore be more robust in terms of finding the correct optimal solution than during low irradiance. To have an impact during higher irradiance, the cyber disturbances must be more significant, which is studied in with the second cyber disturbance, where one or two of the measurements have been substituted by zeros.

Substituting measurements by zeros can be interpreted as severe noise in the cyber system. This has been tested by randomly selecting one or two of the measurements used in the optimizer and substituting its value by zero and maintaining original values for all other measurements. As with the previous cyber disturbance under investigation, the system is simulated during the three different operating situations using the cyber-physical simulation platform, and the effects on each situation are evaluated from a network loss perspective, shown in Fig. 11.

The $x$-axis of Fig. 11 represents the simulation number and from simulation 1 to 200 , one of the measurements has been substituted by zero and from simulation 201 to 400, two measurements have been substituted. For each of the three operating situations, the cyber disturbances cause the optimizer to not converge, which is shown as 0 in the ascendingly sorted losses in Fig. 11. In reality, the losses will depend on the backup strategy chosen in case the optimizer does not converge.

Comparing the losses calculated by the optimizer using original and noisy measurements in Fig. 11a, clearly the substitution of zeros for measurement values affect the optimizer calculation results. The impact difference is more severe than in Fig. 10a due to the chance of one or two of the load point consumption levels being observed as zero, thereby lowering the required power flow.

Comparing the results for each of the three operating situations in Fig. 11a, b and c, the cyber disturbance has larger impact during the second and third operating situations, because the disturbances in some occasions are large enough to divert the optimizer from the global minimum and instead commands more inductive power factor setpoints from the PV plants according to a local minimum. As the irradiance is high during these operating situations, the suboptimal control of the power factor will have a large 


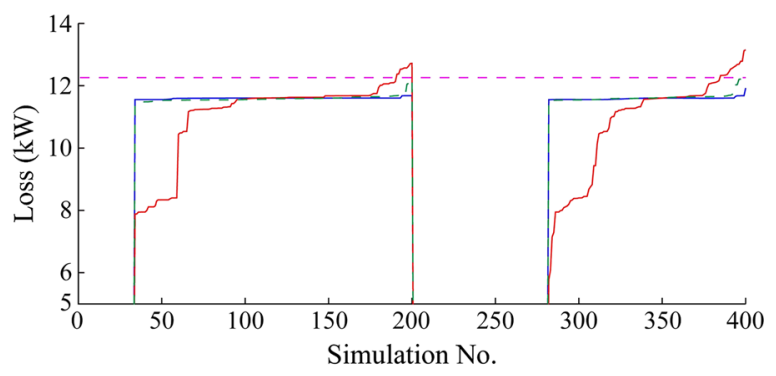

(a) Situation 1: 60\% consumption and $200 \mathrm{~W} / \mathrm{m}^{2}$ irradiance

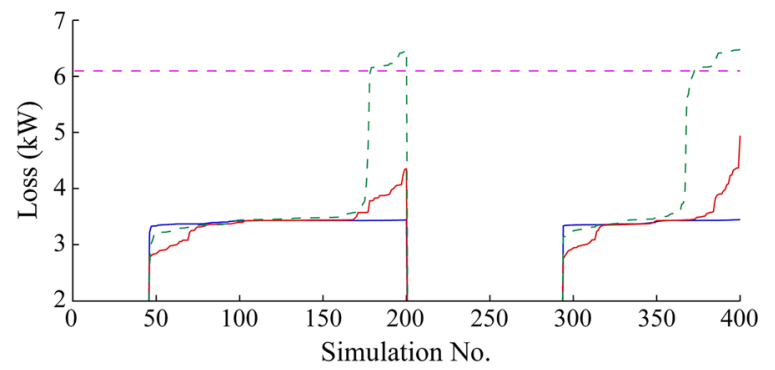

(b) Situation 2: 20\% consumption and $900 \mathrm{~W} / \mathrm{m}^{2}$ irradiance

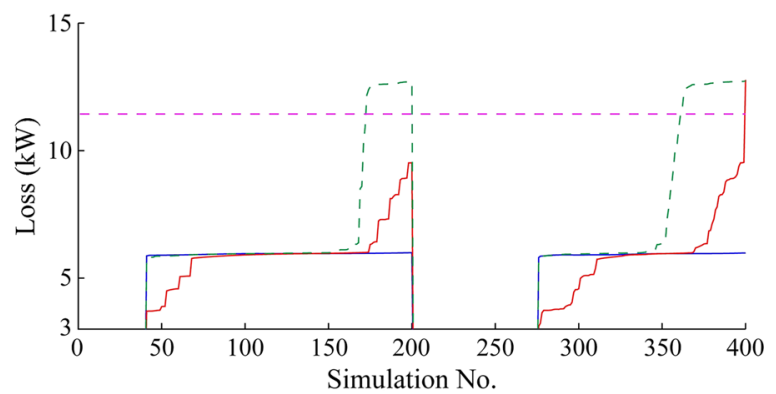

(c) Situation 3: 70\% consumption and $800 \mathrm{~W} / \mathrm{m}^{2}$ irradiance

— Optimizer (original); — Optimizer (noise); - - - RTDS; - - - Local control

Fig. 11 Effects of large noise in the measurement acquisition channel during three different operating situations with centralized reactive power control

effect on the system losses, visible from the RTDS acquired losses in Fig. 11b and c.

Besides noise in the measurement acquisition channel, the effect on the centralized control strategy is evaluated by exposing the distribution of command signals, i.e. power factor set-points, to noise of varying standard deviation.

The system is simulated for each of the three operating situations, represented by the blue, green and red parts of the lines in Fig. 12. A noise standard deviation of $0.5 \%$ is assumed in the simulations 1 to 200,601 to 800 and 1201 to 1400 . For simulations from 201 to 400,801 to 1000 and 1401 to 1600 , the standard deviation of the noise is assumed $1 \%$ and for the rest of the simulation results, the noise standard deviation is assumed $2 \%$.

From the network losses, in Fig. 12a, the cyber disturbance on the power factor set-points clearly has an impact,

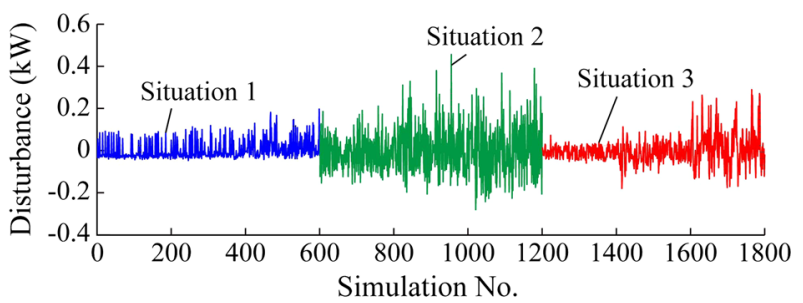

(a) Network loss disturbance

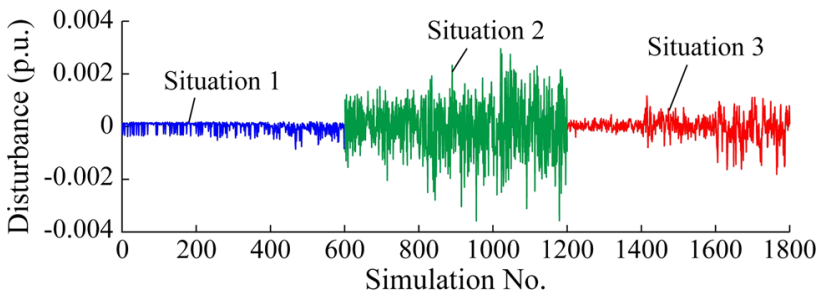

(b) Bus R18 voltage disturbance

Fig. 12 Results from simulating cyber disturbance between optimizer and PV plants for three situations

and most so for the second operating situation. This is due to the high percentage of PV system generation compared to load consumption in this scenario, which increases the influence of the reactive power control of the PV systems in the network. The effects of the set-point noise also affects the voltage magnitude of the nodes in the system, most so for the node furthest away from the feeder, i.e. node R18, as its voltage is affected by the error from all the prior feeders.

The voltage magnitude disturbance of node R18 is visible in Fig. 12b for all the three operating situations, from which the impact can be considered only marginal. However, as the noise magnitude increases, so will the disturbance. The vulnerability of the voltage in the considered operating situations is not close enough to the upper limit of 1.1 p.u. to experience overvoltage, as the maximum voltage is experienced in the second operating situation equal to 1.09 p.u.

In this paper we considered full observability, which means there are sufficient measurements from the system, and perhaps the effect is not pronounced due to this, or the simplicity of the algorithm for centralized control itself. This study however shows that the cyber system can affect the operation of the LV feeder, specifically as disturbances in the system losses and the node voltage magnitudes. Furthermore, the cyber system disturbance simulation test cases show the importance of considering a backup strategy for the centralized control in case the optimizer does not converge. 


\section{Conclusion}

Integration of photovoltaic plants in the low voltage level of the distribution network entails security challenges. One of these issues is high voltage levels at feeder end nodes. This however can be handled by control of the inverters and their reactive power transfer through the utilization of local control strategies. In current literature however, researchers propose using centralized control strategies to optimize the operation of the network and to fulfill an overall objective.

While centralized control improves utilization of the network infrastructure, it simultaneously pushes the network operation towards more vulnerable regions. Combined with the dependency of information and communication technology, centralized control can cause hazardous operation during changing operating situations.

Therefore, this paper has proposed an investigation of boundaries in the irradiance-consumption plane of a low voltage residential feeder with high share of photovoltaics. The evaluation of the two control strategies is done based on simulations conducted in an established cyber-physical simulation platform. The boundaries for coordinating between local and centralized control have been found for a specific feeder during normal operation and while considering perturbations in the physical system, i.e. changing consumption and irradiance.

The identified boundaries can assist in the decisionmaking process of choosing a suitable reactive power control strategy for the photovoltaic plants during different operational situations.

With the representation of the cyber system, simulation platform was used to evaluate the effects from cyber disturbances on the centralized voltage regulation strategy. During small magnitude noise in the data acquisition channel, network losses were affected while operating in medium consumption and low irradiance conditions. Furthermore, the system was susceptible to large cyber disturbances. During all three considered operating situations, the optimizer was unable to converge at the global minimum, or at all in some occasions.

Possible areas of future work include, removing the assumption of full observability and evaluate different cyber system designs to assist in forming the control strategy coordination. Furthermore, evaluating the effects of physical and cyber system disturbances on the cyber system performance, and generalization of the boundary identification for coordinating the control strategies could be considered.

Acknowledgements This work is supported by Danmarks Tekniske Universitet.
Open Access This article is distributed under the terms of the Creative Commons Attribution 4.0 International License (http:// creativecommons.org/licenses/by/4.0/), which permits unrestricted use, distribution, and reproduction in any medium, provided you give appropriate credit to the original author(s) and the source, provide a link to the Creative Commons license, and indicate if changes were made.

\section{References}

[1] Yang G, Marra F, Juamperez M (2015) Voltage rise mitigation for solar PV integration at LV grids. J Mod Power Syst Clean Energy 3(3):411-421

[2] Tahir M, Nassar ME, El-Shatshat R et al (2016) A review of volt/var control techniques in passive and active power distribution networks. In: Proceedings of the 2016 IEEE smart energy grid engineering, Oshawa, Canada, 21-24 August 2016, 7 pp

[3] BDEV (2008) Technical guidelines: Generating plants connected to the medium-voltage network

[4] Energinet (2016) Technical regulation 3.2.2 for PV power plants above $11 \mathrm{~kW}$. Energinet, Fredericia

[5] Juamperez M, Yang G, Kjær SB (2014) Voltage regulation in LV grids by coordinated volt-var control strategies. J Mod Power Syst Clean Energy 2(4):319-328

[6] Karagiannopolous S, Aristidou P, Hug G (2017) Hybrid approach for planning and operating active distribution grids. J IET Gener Transm Dis 11(3):685-695

[7] Vovos PN, Kiprakis AE, Wallace AR et al (2007) Centralized and distributed voltage control: Impact on distributed generation penetration. IEEE Trans Power Syst 22(1):476-483

[8] Turitsyn K, Sulc P, Backhaus S et al (2011) Options for control of reactive power by distributed photovoltaic generators. Proc IEEE 99(6):1063-1073

[9] Isozaki Y, Yoshizawa S, Fujimoto Y et al (2016) Detection of cyber attacks against voltage control in distribution power grids with PVs. IEEE Trans Smart Grid 7(4):1824-1835

[10] Yu X, Xue Y (2016) Smart grids: a cyber-physical systems perspective. Proc the IEEE 104(5):1058-1070

[11] Hahn A, Ashok A, Sridhar S et al (2013) Cyber-physical security testbeds: architecture, application and evaluation for smart grids. IEEE Trans Smart Grid 4(2):847-855

[12] CIGRÉ (2014) Benchmark systems for network integration of renewable and distributed energy resources. CIGRÉ Task Force C6.04.02

[13] Singhal A, Ajjarapu V, Fuller JC et al (2017) Real-time local volt/var control under external disturbances with high PV penetration. IEEE Transactions on Smart Grid. https://doi.org/10. 1109/TSG.2018.2840965

[14] Zhao J, Li Y, Li P et al (2017) Local voltage control strategy of active distribution network with PV reactive power optimization. In: Proceedings of the 2017 IEEE PES general meeting, Chicago, USA, 16-20 July 2017, 5 pp

[15] Zhang C, Xu Y, Dong ZY et al (2017) Three-stage robust inverter-based voltage var control for distribution networks with high-level PV. IEEE Trans Smart Grid. https://doi.org/10.1109/ TSG.2017.2752234

[16] Cintuglu MH, Mohammed OA, Akkaya K et al (2017) A survey on smart grid cyber-physical system testbeds. IEEE Commun Surv Tut 19(1):446-464

[17] Venkataramanan V, Srivastava A, Hahn A (2016) Real-time sco-simulation testbed for microgrid cyber-physical analysis. In: Procceedings of the 2016 workshop on modeling and simulation 
of cyber-physical energy systems, Vienna, Austria, 11 April 2016, 6 pp

[18] Chen B, Butler-Purry KL, Kundur D (2014) Implementing a real-time cyber-physical system test bed in RTDS and OPNET. In: Proceedings of the 2014 north American power symposium, Pullman, USA, 7-9 September 2014, 6 pp

[19] RTDS (2014) Connecting RSCAD/Runtime with external applications. RTDS software documentation

[20] Moyne JR, Tilbury DM (2007) The emergence of industrial control networks for manufacturing control, diagnostics, and safety data. Proc IEEE 95(1):29-47

[21] Thomas MS, McDonald JD (2015) Power system SCADA and smart grids. CRC Press, Boca Raton

[22] Ideal Grid for All (2015) Deliverable 5.1: State estimation and forecasting algorithms on MV and LV networks. http://www.tut. fi/eee/ide41/IDE4L-WP5-D5.1-\%20V\%201.0.pdf. Accessed 27 Nov 2017

[23] Ranamuka D, Agalgaonkar AP, Muttaqi KM (2014) Online voltage control in distribution systems with multiple voltage regulating devices. IEEE Trans Sustain Energ 5(2):617-628

[24] Bailey D, Wright E (2003) Practical SCADA for industry. Newnes, UK

[25] Falahati B, Fu Y (2014) Faults and failures in cyber-power interdependent networks. In: Proceedings of the 2014 IEEE PES T\&D conference and exposition, Chicago, USA, 14-17 April 2014, 5 pp

[26] Drayer E, Hegemann J, Gehler S et al (2016) Resilient distribution grids-cyber threat scenarios and test environment. In: Proceedings of the 2016 IEEE PES innovative smart grid technologies conference Europe, Ljubljana, Slovenia, 9-12 October 2016, 6 pp

[27] Panteli M, Kirschen DS (2011) Assessing the effect of failures in the information and communication infrastructure on power system reliability. In: Proceedings of the 2011 IEEE PES power system conference and exposition, Phoenix, USA, 20-23 March 2011, $7 \mathrm{pp}$

[28] HelioClim-3 Archives (2017) Solar radiation data, Sophia Antipolis, France. http://www.soda-pro.com/web-services/ radiation/helioclim-3-archives-for-free. Accessed 27 Oct 2017

[29] Profiler 2012 (2017) Elforbrugs panelerne, http://www. elforbrugspanel.dk/Pages/Rapportering.aspx\#ID18. Accessed 26 Oct 2017

[30] Repo S, Ponci F, Giustina DD et al (2017) The IDE4L project: defining, designing, and demonstrating the ideal grid for all. IEEE Power and Energy Magazine 15(3):41-51
Theis Bo RASMUSSEN received the B.Sc. and M.Sc. degree in Electrical Engineering, with specialization in Electric Energy Systems, from the Technical University of Denmark, Kgs. Lyngby, Denmark, in 2014 and 2016, respectively. Currently he is pursuing a Ph.D. degree at the Center for Electric Power and Energy, Department of Electrical Engineering, Technical University of Denmark, Kgs. Lyngby, Denmark. His research interests include cyber-physical systems, simulation technology and integration of renewables.

Guangya YANG received the B.E., M.E., and Ph.D. degrees all in the field of electric power system, in 2002, 2005, and 2008, respectively. $\mathrm{He}$ is currently an Associate Professor with the Center for Electric Power and Energy, Department of Electrical Engineering, Technical University of Denmark. He has been leading various industrial collaborative projects in Denmark in the field of monitoring, operation and protection of renewable energy systems. He is editor of IEEE Transactions on Sustainable Energy. His research interests include renewable energy integration, security and control of low inertia systems, and cyber-physical energy systems.

Arne Hejde NIELSEN is an Associate Professor at Centre of Electric Technology, DTU Electrical Engineering. He has 30 years experience in electric power engineering; the first years from ASEA AB, Central Research and Development Department, Sweden working with measurement technology and motor design and control. During the latest 15 years, his focus has been on electric power systems especially on implementation of renewable energy sources in the power system.

Zhaoyang DONG obtained his Ph.D. from the University of Sydney, Australia in 1999. He is now Professor of energy systems at the University of NSW, Sydney, Australia. His immediate role was Professor and Head of School of Electrical and Information Engineering, the University of Sydney. He also worked as manager for system planning with Transend Networks (TAS Networks now), Australia for transmission network planning. His research interests include smart grid, power system planning, power system security assessment, load modeling, microgrid, electricity market, and computational intelligence and its application in power engineering. He is an editor of IEEE Transactions on Smart Grid, IEEE PES Letters and IET Renewable Power Generation. He is a Fellow of IEEE. 